\title{
Spermatic Cord Anastomotic Leakage
}

National Cancer Institute

\section{Source}

National Cancer Institute. Spermatic Cord Anastomotic Leakage. NCI Thesaurus. Code C78622.

Leakage due to breakdown of a spermatic cord anastomosis. 